\title{
Theoretically speaking
}

\section{Are true theorists a dying breed? Does more need to be done to support and encourage young scientists to devote themselves to inventing new theoretical concepts and models?}

Although it's undoubtedly true that the majority of papers published in Nature Photonics are of an experimental nature, the value, impact and influence of theoretical studies should never be underestimated. Over the years, new concepts have played a pivotal role in developing the field of photonics, right from Maxwell's formulation of his equations of electromagnetism (celebrated in our January issue earlier this year) through to the concept of the laser, the optical fibre and metamaterials, to name just a few breakthroughs. There's no doubt that new concepts can have a significant influence but, interestingly, there's often a long timelag before experimental capabilities allow ideas to be realized and put to good use. The generation of electromagnetic waves did not take place until many years after Maxwell first predicted their existence, optical fibres were not a realistic proposition until high-purity glass was developed and metamaterials, especially those operating at optical frequencies, required advances in nanofabrication before they could be fabricated. It can take decades for technological progress to catch up with theory and allow new hypotheses to be experimentally tested. For example, it took more than twenty years to experimentally observe lasing from quantum cascade lasers even though the key idea of intersubband light amplification in quantum wells electrically pumped by resonant tunnelling was theoretically proposed by Kazarinov and Suris in 1971.

So what makes a theoretical paper well-suited for Nature Photonics? Well, to start, we don't want simulations or numerical calculations that simply confirm a well-known phenomenon or optimize an optical device. Instead, we're far more interested in new concepts or insights that led to surprising or unexpected behaviour or effects, especially if the findings have the potential to ultimately lead to the development of devices with unique functionality or a fundamental new way of thinking about the behaviour of light.

The question of whether some form of experimental evidence is required to substantiate a theoretical paper is an interesting and debatable topic. In many areas of physics such evidence can be very hard to gather at the inception of the idea - just take the detection of the Higgs Boson or gravitational waves, as examples. The former was only recently found despite being predicted in the 1960s and the latter is still being searched for by telescopes and giant interferometers around the world. In such cases, it would clearly be unreasonable and unrealistic to expect an experimental proof of such phenomena at the outset. However, when a theory paper concerns a new device design, an assessment of the feasibility of the idea and its prospects for realization do reflect on the impact of the study, and we feel the need to take that into account.

\section{The meaning of 'theoretician' has evolved dramatically and currently many people call themselves theoreticians while only solving known equations numerically.}

Although, as history shows, streams of new science are often stirred up and inspired by theory papers, the current role of many theoreticians has changed significantly and is continuing to evolve. To obtain competitive funds from national or international research funding agencies, many theoreticians now work far more closely with experimentalists than ever before.

Leading-edge research explores such complex subjects that experimentalists are no longer able to do theoretical analysis by themselves on the side. Theoreticians increasingly perform analysis to extract meaningful information from experimental results. Sometimes, they suggest an additional experiment that would allow experimentalists to clearly support their theory. Modern theoreticians have increasingly become multitaskers and an indispensable aid to experimentalists.

The validation of theoretical works is given by experimental results, not by subsequent theoretical works. As such, traditional theoreticians have to be patient until experimentalists attempt to perform verification experiments. However, there are some theoreticians who perform experiments by themselves to validate their theory if experimentalists are not motivated to do so. Yaron Silberberg of the Weizmann Institute of Science told Nature Photonics: "You see more and more theoreticians who want to run a small lab of their own, and experimentalists who make a serious theory effort, or at least have theory done as an integral part of their group."

However, there are some theoreticians who have concerns about the evolving role of theorists, especially if it means that too many become committed to conducting numerical calculations for theoretical analysis, rather than investing time and energy in developing new conceptual models.

“The meaning of 'theoretician' evolved dramatically since my undergraduate days and currently many people call themselves theoreticians while only solving known equations numerically," explains Yuri Kivshar of the Australian National University. According to Kivshar, good theory papers are of two major classes: (i) a prediction of a new effect and (ii) a prediction of a new concept that may generate a new stream of research.

In particular, he's worried that not enough is being done to cultivate and train young theoreticians and encourage them to devote themselves to such studies. In many ways, this is hardly surprising as there are many possible hurdles to be overcome for those wishing to devote themselves to developing new theories. The merit system within academia doesn't always help. It takes great time and effort to develop a new theory. However, to obtain funding, or to obtain tenure, there is great pressure on scientists to publish an increasing volume of papers and demonstrate the short-term applications of their work. To this end, some theoreticians are apt to publish papers reporting numerical simulations based on well-known models and using commercial mathematical software, even though such papers have little or no conceptual value. The environment of academic research seems challenging, in a sense, for genuine theoreticians. Perhaps more needs to be done to help foster such endeavours of the mind? 